\title{
Stroke in young secondary to infective endocarditis complicating hypertrophic cardiomyopathy - A case report
}

\author{
Abhishek Bhandari, Bhupendra Shah, Aditya Mahaseth, Sanjib Kumar Sharma \\ Department of Internal Medicine, B. P. Koirala Institute of Health Sciences, Dharan- 18, Nepal \\ Corresponding Author: Abhishek Bhandari \\ Department of Internal Medicine, B. P. Koirala Institute of Health Sciences, Dharan, Nepal \\ E-mail: bhandari_sxc@hotmail.com
}

Cite this article as: Bhandari A, Shah B, Mahaseth A, et al. Stroke in young secondary to infective endocarditis complicating hypertrophic cardiomyopathy - A case report. Nepalese Heart Journal 2019; Vol 16(1), 57-59

Received date: 6th April 2018

Accepted date: 19th September 2018

\begin{abstract}
Hypertrophic cardiomyopathy is an autosomal dominant disease of cardiac sarcomere with the varied clinical presentation. The common manifestations are heart failure (dyspnea), palpitations, chest pain, stroke and even sudden death. Majority of patients are not recognized because of being asymptomatic. Stroke is one of the complications of hypertrophic cardiomyopathy and usually occurs in the setting of atrial fibrillation, advanced age and congestive cardiac failure. Although infective endocarditis is known, but relatively uncommon, complication of hypertrophic cardiomyopathy, it is not reported from Nepal. We report here a case of 42 -year male patient with hypertrophic cardiomyopathy in normal sinus rhythm with infective endocarditis who developed malignant cardioembolic stroke involving left middle cerebral artery territory.
\end{abstract}

Keywords: Anticoagulation, hypertrophic cardiomyopathy, infective endocarditis, stroke

DOI: https://doi.org/10.3126/njh.v16i1.23904

\section{Introduction}

Stroke is the commonest cause of adult disability and second most common cause of mortality worldwide. ${ }^{1}$ Ischemic stroke consists of $90 \%$ of total stroke ${ }^{2}$ and cardioembolic stroke constitutes around $20 \%$ of ischemic stroke. ${ }^{3}$ The common risk factors for cardioembolic stroke are non-rheumatic valvular atrial fibrillation, myocardial infarction, prosthetic valves, rheumatic heart disease and ischemic cardiomyopathy. ${ }^{3}$ Stroke and systemic embolic events were the known complications of hypertrophic cardiomyopathy which occurred commonly in patients with accompanying atrial fibrillation. ${ }^{4}$ However, stroke in hypertrophic cardiomyopathy with normal sinus rhythm is a rare phenomenon. Our patient was a 42 -year-old right handed smoker with no significant past medical history who presented with fever of 2 weeks duration without localizing symptoms and signs. Cardiovascular examination along with the presence of pandigital clubbing pointed the possibility of IE and was admitted. During the hospital stay, the patient developed sudden onset right-sided hemiparesis with a reduced level of consciousness and aphasia suggestive of cardio-embolic stroke in view of hypertrophic cardiomyopathy with infective endocarditis.

\section{Case Report}

A 42-year-old patient, right-handed, smoker, a shopkeeper by occupation and asymptomatic for other medical illness presented with a complaint of intermittent high-grade fever with chills and rigors for 2 weeks without any localizing symptoms. The patient had no significant past medical history or recreational drug use. Patient's father had a history of paroxysmal supraventricular tachycardia and was taking calcium channel blocker.

On out-patient room, the patient had a pulse rate of $83 / \mathrm{min}$, regular in rhythm, blood pressure of $120 / 70 \mathrm{~mm} \mathrm{Hg}$, respiratory rate of $20 / \mathrm{min}$ and temperature of $98.40^{\circ} \mathrm{F}$. The patient had pandigital clubbing on examination. On cardiac examination, the patient had normally placed apical impulse with grade II ejection systolic murmur at apex without radiation to neck or axilla. Other systemic examinations were unremarkable. On lab reports, hemoglobin- $12.7 \mathrm{~g} / \mathrm{dl}$, total leukocyte count- 14900/ $\mathrm{mm}^{3}$ and platelets count- $256000 / \mathrm{mm}^{3}$. Blood cultures (3 sets sent from 3 different sites) were positive for Enterococcus species. Electrocardiogram showed nonspecific repolarization abnormalities. Transthoracic echocardiogram (TTE) revealed

@Nepalese Heart Journal. Nepalese Heart Journal retains copyright and work is simultaneously licensed under Creative Commons Attribution License CC - BY 4.0 that allows others to share the work with an acknowledgement of the work's authorship and initial publication in this journal. 
asymmetric septal hypertrophy with resting left ventricular outflow gradient of $30 \mathrm{~mm} \mathrm{Hg}$ with the left atrial size of $36 \mathrm{~mm}$ diameter and no intracardiac thrombus or vegetations. Systolic anterior motion of anterior mitral leaflet was not seen. (Figure 1). We made the diagnosis of hypertrophic cardiomyopathy with infective endocarditis and treated with injectable antibiotics and beta blocker.

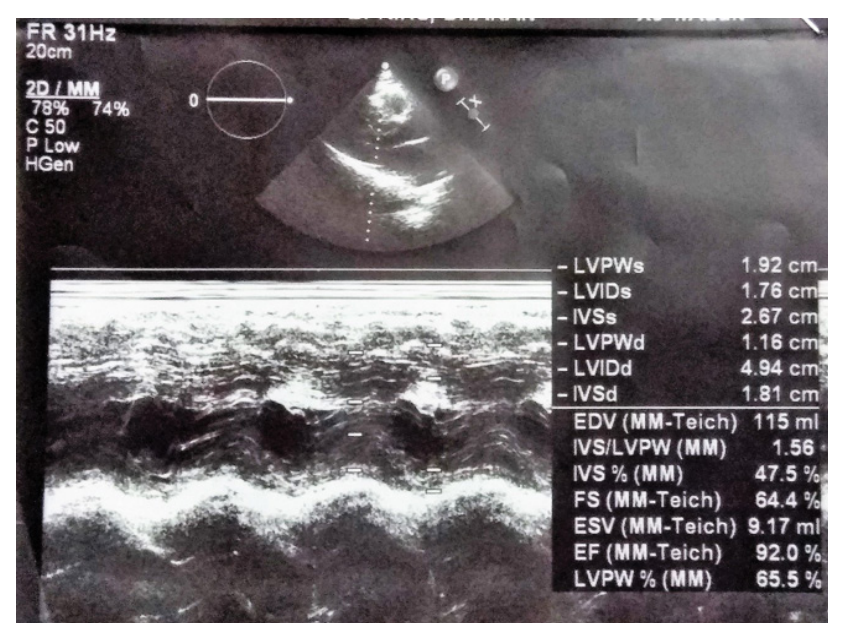

Figure 1: Echocardiogram of a patient with stroke secondary to infective endocarditis complicating hypertrophic cardiomyopathy

On the second day of admission, the patient had sudden onset weakness of right half of body with decreased consciousness level with Glasgow coma scale score of $8 / 15$. On motor examination the power was $0 / 5$ on all range of motion of small joints of hands and feet, ankle, knee, hip, wrist, elbow and shoulder joint on the right side. The patient had global aphasia and right plantar was upgoing, had lateral rectus palsy on the left side with the normal reactive pupil on both side. Computed tomography scan of the head revealed a large infarct in the left fronto-temporal-parietal region involving external capsule, internal capsule (posterior limb) and left lentiform nucleus (left middle cerebral artery territory) with mass effect on the adjacent structures. (Figure 2). Diagnosis of ischemic left sided cerebrovascular accident involving left middle cerebral artery territory with features of raised intracranial pressure with modified Rankin Scale of five was made. The patient received intravenous mannitol for raised intracranial pressure. During critical care stay, 48-hour continuous telemetric electrocardiogram did not reveal any ventricular or atrial tachyarrhythmias. Gradually signs of raised intracranial pressure disappeared and the patient remained under a multi-disciplinary rehabilitation program. Further blood investigations revealed fasting blood sugar of $98 \mathrm{mg} / \mathrm{dl}, 2$ hour post prandial $137 \mathrm{mg} / \mathrm{dl}$, total cholesterol of $151 \mathrm{mg} / \mathrm{dl}$, LDL of $88 \mathrm{mg} / \mathrm{dl}$, HDL of $45 \mathrm{mg} / \mathrm{dl}$ and triglycerides of $93 \mathrm{mg} / \mathrm{dl}$.

Follow up after six weeks revealed improve Glasgow Coma Scale with functional status of Modified Rankin Scale of four. Oral intake was adequate. Power in his right lower limb improved to $2 / 5$ on hip flexors and extensors with no improvement in upper limb. He was able to understand instructions given to him though could not produce speech. He had no episodes of aspiration and no bed sores. He is continuing oral medications and physiotherapy as prescribed.

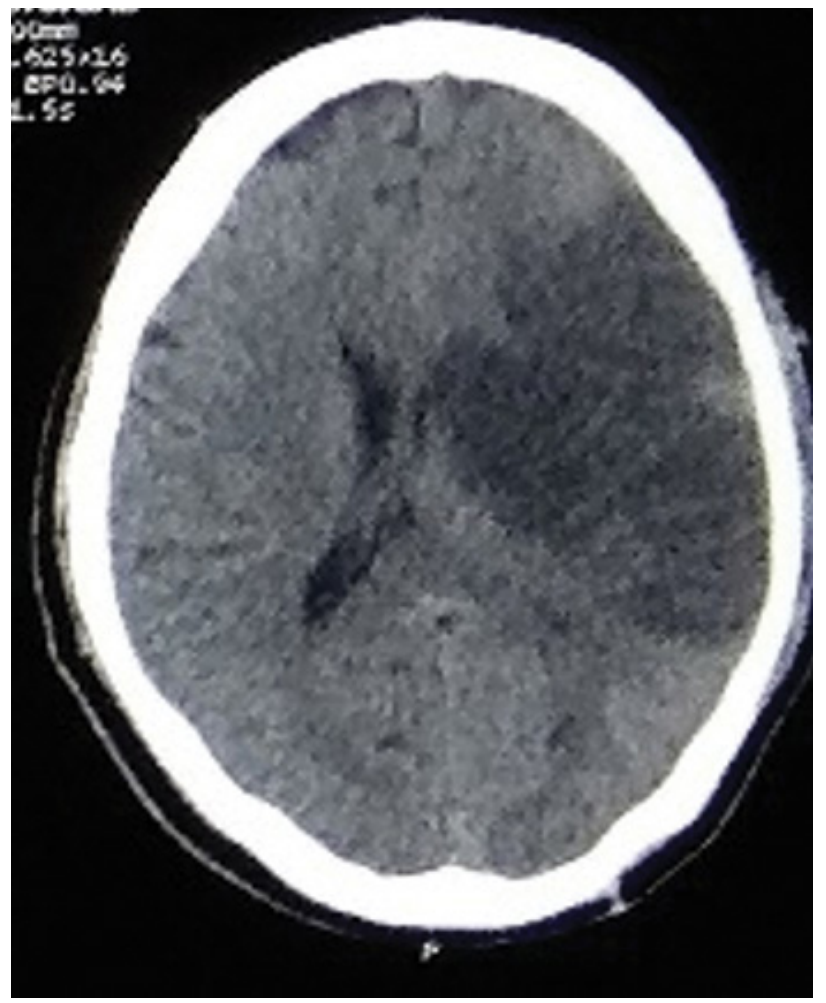

Figure 2: Computed Tomography of Head

\section{Discussion}

The prevalence of hypertrophic cardiomyopathy is 1 in 500 adults in the general population. ${ }^{4}$ It is characterized by asymmetric hypertrophy of ventricular septum with or without the mechanism of left ventricular outflow tract obstruction. Hypertrophic cardiomyopathy may be associated with mitral regurgitation, diastolic dysfunction, myocardial ischemia and cardiac arrhythmias (ventricular arrhythmias and atrial fibrillation). ${ }^{5}$ The common presentations of hypertrophic cardiomyopathy are asymptomatic presentation (detected on routine examination), dyspnea, atrial fibrillation (20\%), and anginal chest pain. ${ }^{6}$

Cardioembolic stroke in a hypertrophic cardiomyopathy occurs in the setting of chronic or paroxysmal atrial fibrillation. ${ }^{7}$ Maron et al in community-based cohort study showed the prevalence of stroke and peripheral arterial embolization was $6 \%$ among hypertrophic cardiomyopathy patients. These complications were more common in the elderly, New York Heart Association class III-IV patients, occurred almost exclusively in patients with paroxysmal or chronic atrial fibrillation and found to be reduced in frequency by anticoagulation. ${ }^{7}$ In contrast to this finding, our patient had a stroke without documented atrial fibrillation. The current guidelines recommend the use of anticoagulants in a patient with hypertrophic cardiomyopathy with atrial fibrillation. ${ }^{8}$ However, the evidence to use anticoagulant to prevent cardioembolic stroke in hypertrophic cardiomyopathy with normal sinus rhythm is not known. Haruki et al (2016) in hospital-based observational study demonstrated older age at diagnosis and enlarged left atrial dimension of more than 47 $\mathrm{mm}$ were the possible risk factors for embolic events in patients with hypertrophic cardiomyopathy without documented atrial fibrillation. ${ }^{9}$ However, our patient had normal size left atrium (36mm).

The incidence of infective endocarditis among hypertrophic 
cardiomyopathy patients was 18 to 28 times higher than that of the general population. ${ }^{10}$ Our patient had blood culture positive for Enterococci and clubbing was present even though there were no other peripheral signs of infective endocarditis. We suspect that stroke in our patient occurred due to dislodgement of the vegetation of infective endocarditis, though TTE did not reveal vegetation. However, due to unavailability at the time, transesophageal echochardiogram was not performed in our patient.

This case report highlights the focused clinical examinations to identify the possible source of fever as early detection and management of IE might have prevented the catastrophic event.

\section{Conclusion}

Hypertrophic cardiomyopathy is a risk factor for cardioembolic stroke in young. The predictors of stroke in hypertrophic cardiomyopathy are atrial fibrillation, enlarged size of left atrium, elderly age. However, stroke can occur even in the setting of normal sinus rhythm, normal left atrial size and a young patient. Presence of fever in a patient with hypertrophic cardiomyopathy warrants investigation for IE and close observation for possible systemic embolization.

\section{Acknowledgement}

We are very thankful to the patient and his wife for allowing us to share his condition to contribute to science.

\section{References}

1. Bennett DA. The global burden of ischemic stroke: findings of the GBD 2010 study. Global heart. 2014 Mar $31 ; 9(1): 107-12$

https://doi.org/10.1016/j.gheart.2014.01.001

2. Andersen KK, Olsen TS, DehlendorffC, et al. Hemorrhagic and ischemic strokes compared: stroke severity, mortality, and risk factors. Stroke. 2009 Jun 1;40(6):2068-72. https://doi.org/10.1161/STROKEAHA.108.540112

3. Leary MC, Caplan LR. Cardioembolic stroke: An update on etiology, diagnosis and management. Annals of Indian Academy of Neurology. 2008 Jan 1;11(5):52.

4. Maron BJ. Prevalence of Hypertrophic cardiomyopathy in a general population of young adults. Circulation. $1995 \mathrm{Aug}$ $15 ; 92(4): 785-9$ https://doi.org/10.1161/01.CIR.92.4.785

5. Nishimura RA, Holmes Jr DR. Hypertrophic obstructive cardiomyopathy. New England Journal of Medicine. 2004 Mar 25;350(13):1320-7. https://doi.org/10.1056/NEJMcp030779

6. Houston BA, Stevens GR. Hypertrophic cardiomyopathy: a review. Clinical Medicine Insights. Cardiology. 2014;8(Suppl 1):53.

https://doi.org/10.4137/CMC.S15717

7. Maron BJ. Clinical profile of stroke in 900 patients with hypertrophic cardiomyopathy. Journal of the American College of Cardiology. 2002 Jan 16;39(2):301-7. https://doi.org/10.1016/S0735-1097(01)01727-2

8. MacIntyre C, Lakdawala NK. Management of atrial fibrillation in hypertrophic cardiomyopathy. Circulation. 2016 May 10;133(19):1901-5.

https://doi.org/10.1161/circulationaha.115.015085
9. Haruki S, Minami Y, Hagiwara N. Stroke and Embolic Events in Hypertrophic Cardiomyopathy. Stroke. 2016 Apr 1;47(4):936-42. https://doi.org/10.1161/STROKEAHA.115.012130

10. Spirito P. Infective endocarditis in hypertrophic cardiomyopathy. Circulation. 1999 Apr 27;99(16):2132-7. https://doi.org/10.1161/01.CIR.99.16.2132 